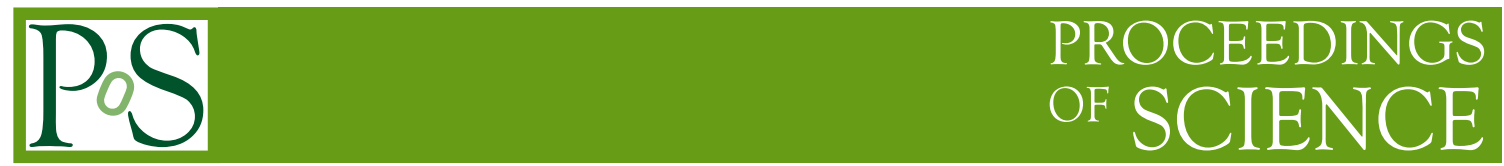

\title{
Quarkonium suppression from parton energy loss
}

\author{
Stéphane Peigné \\ SUBATECH, Nantes \\ E-mail: peigneesubatech.in2p3.fr
}

The effects of energy loss in cold nuclear matter on $J / \psi$ suppression in p-A collisions are studied. A simple model based on first principles (gluon radiation associated to a hard process) and depending on a single free parameter (the rate per unit length of transverse momentum broadening $\hat{q}$ ) is able to reproduce $J / \psi$ suppression data at large $x_{F}$ or large rapidity and at various center-of-mass energies. These results strongly support parton energy loss as a dominant effect in quarkonium suppression. It is also observed that at large rapidity, saturation effects should be less important than energy loss, even at LHC energies. Predictions of the parton energy loss picture for $J / \psi$ and $\Upsilon$ suppression in $\mathrm{p}-\mathrm{Pb}$ collisions at the LHC are made.

Sixth International Conference on Quarks and Nuclear Physics

April 16-20, 2012

Ecole Polytechnique, Palaiseau, Paris 


\section{General context}

The spectacular nuclear suppression (jet-quenching) of hadrons produced at large $p_{T}$ in $\mathrm{Pb}-\mathrm{Pb}$ collisions at the LHC (see e.g. $[1,2]$ ) finds a natural explanation in terms of parton energy loss in a quark-gluon plasma (QGP). For light hadron production at mid-rapidity and sufficiently large $p_{T}$, the parton energy loss is dominantly radiative, in average of the form $\Delta E \sim \alpha_{s} \hat{q} L^{2}$ [3], with $L$ the distance travelled by the parton through the medium and $\hat{q}$ the rate per unit length of transverse momentum broadening in the medium. The strength of jet-quenching can be explained if $\hat{q}$ in the QGP is larger, by one to two orders of magnitude, than its expected value in cold nuclear matter $\hat{q}_{0} \sim 0.05 \mathrm{GeV}^{2} / \mathrm{fm}$.

Drastic nuclear suppression effects are not only seen in A-A but also in $\mathrm{p}-\mathrm{A}$ collisions, for instance for quarkonium [4] but also light hadron [5] production at large $x_{F}$ (or large rapidity). Understanding nuclear suppression in cold nuclear matter should be a prerequisite in order to interpret quantitatively nuclear suppression in heavy-ion collisions. However, it is striking that there is no consensus yet on the origin of $J / \psi$ suppression at large rapidity $/ x_{F}$ in $\mathrm{p}-\mathrm{A}$ collisions, from SPS to RHIC [6, 4, 7], despite many theoretical attempts (see [8] for a review).

Recently, new scaling properties have been identified for the induced gluon radiation spectrum $\mathrm{d} I / \mathrm{d} \omega$, and associated energy loss $\Delta E$, of certain hard processes [9]. The phenomenological consequences of these results on $J / \psi$ and $\Upsilon$ nuclear suppression in $\mathrm{p}-\mathrm{A}$ and $\pi$-A collisions have then been studied [10]. In this talk I present the main idea and results of Refs. $[9,10]$.

I will show that the large $x_{F}$ quarkonium suppression data in $\mathrm{p}-\mathrm{A}$ collisions, parametrized by

$$
R_{\mathrm{pA}}^{\psi}\left(x_{F}, \sqrt{s}\right)=\frac{1}{A} \frac{\mathrm{d} \sigma_{\mathrm{pA}}^{\psi}}{\mathrm{d} x_{F}}\left(x_{F}, \sqrt{s}\right) / \frac{\mathrm{d} \sigma_{\mathrm{pp}}^{\psi}}{\mathrm{d} x_{F}}\left(x_{F}, \sqrt{s}\right),
$$

can be explained by parton energy loss in cold matter. Although $\hat{q}$ in cold matter is small, a strong nuclear attenuation arises due to the specific parametric behaviour $\Delta E \propto E$ at sufficiently large $E$, where $E$ is the quarkonium energy in the target nucleus rest frame. As discussed in Ref. [9], this behaviour holds when the partonic subprocess can be viewed (in the nucleus rest frame) as the small angle scattering of a color charge. In the following we focus on quarkonium hadroproduction, where the mass $M$ of the heavy-quark $Q \bar{Q}$ pair provides the hard scale.

The physics of $J / \psi$ nuclear suppression depends crucially on the precise kinematics under consideration. When the $J / \psi$ hadronization time $t_{\text {hadro }} \ll L$, with $L$ the nuclear size, nuclear suppression arises from the absorption cross section $\sigma_{a b s}$ of the fully formed $J / \psi$ in the nucleus (see Fig. 1 left). When $t_{\text {hadro }} \gg L$, what propagates through the nucleus is a compact color octet $c \bar{c}$ pair (Fig. 1 right), and $\sigma_{a b s}$ becomes irrelevant. In the following we focus on the latter kinematical
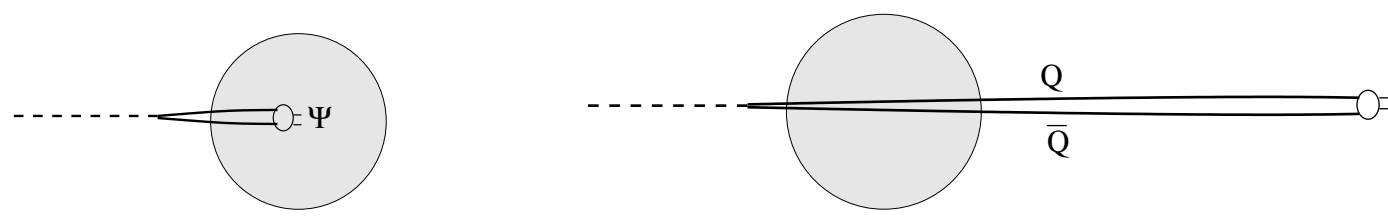

Figure 1: Quarkonium hadroproduction at small (left) and large (right) quarkonium energy $E$ in the nucleus rest frame. In the latter case a compact color octet $Q \bar{Q}$ pair crosses the nucleus. 
domain. In the Figures presented in Section 4, we indicate by an arrow the typical value of $x_{F}$ (or rapidity) where $t_{\text {hadro }}=L$, above which our model should be valid.

\section{Parton energy loss in $J / \psi$ hadroproduction}

The presence of a large (medium-induced) parton energy loss associated to $J / \psi$ hadroproduction can be understood as follows. At large $x_{F}$, the gluon emission required for the $c \bar{c}$ color neutralization is constrained to be softish by energy conservation, and thus the $c \bar{c}$ pair remains color octet for a long time $t_{\text {octet }} \gg t_{\text {hard }}$, where $t_{\text {hard }} \sim E / M^{2}$ is the perturbative production time of the $Q \bar{Q}$ pair. As a consequence, at large $x_{F}$ the $J / \psi$ hadroproduction subprocess looks like small angle scattering of a color charge, as pictured in Fig. 2. The associated radiation spectrum arising from gluon formation times $t_{f}$ satisfying $t_{\text {hard }} \ll t_{f} \ll t_{\text {octet }}$ is easy to calculate [9]. It is similar to the (non-abelian) Bethe-Heitler spectrum of an asymptotic charge, and depends on the amount of transverse momentum kick $q_{\perp}$ to the charge. The typical $q_{\perp}$ is expected to be larger in $\mathrm{p}-\mathrm{A}$ than in p-p collisions due to transverse momentum nuclear broadening $\Delta q_{\perp}^{2}=\hat{q} L$. The resulting medium-induced radiation spectrum is similar to the Bethe-Heitler spectrum with $q_{\perp}^{2}$ replaced by $\Delta q_{\perp}^{2}$ [9], and the average energy loss scales as the quarkonium energy, $\Delta E \propto E$.

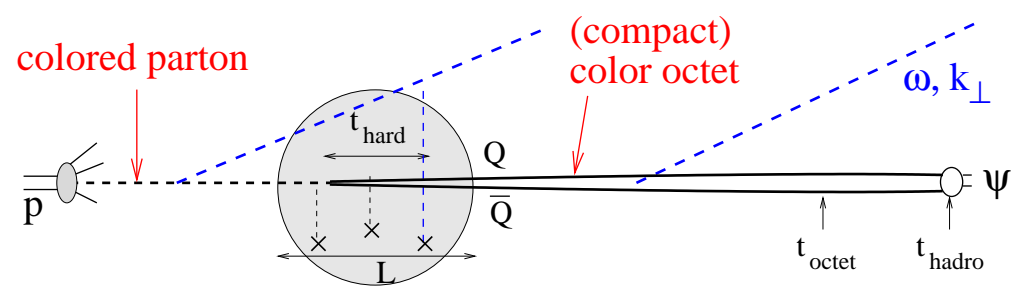

Figure 2: Generic process for $J / \psi$ production in p-A collisions, assuming $L, t_{\text {hard }} \ll t_{\text {octet }} \lesssim t_{\text {hadro }}$. An induced radiated gluon $\left(\omega, \mathbf{k}_{\perp}\right)$ with formation time $t_{f}$ satisfying $L, t_{\text {hard }} \ll t_{f} \ll t_{\text {octet }}$ is represented.

The behaviour $\Delta E \propto E$ for $J / \psi$ hadroproduction originates from coherent radiation, which is neither an initial nor a final state effect. We expect $\Delta E \propto E$ in all situations where an incoming (color) charge is scattered to an outgoing charge, as in open charm and light hadron production, see Fig. 3 (top). However $\Delta E \propto E$ is not expected in DIS nor Drell-Yan production off nuclei (Fig. 3 bottom), where the purely final-state (initial-state) DGLAP-like radiation (with associated $\left.t_{f} \gg t_{\text {hard }}\right)$ cancels in the medium-induced spectrum.
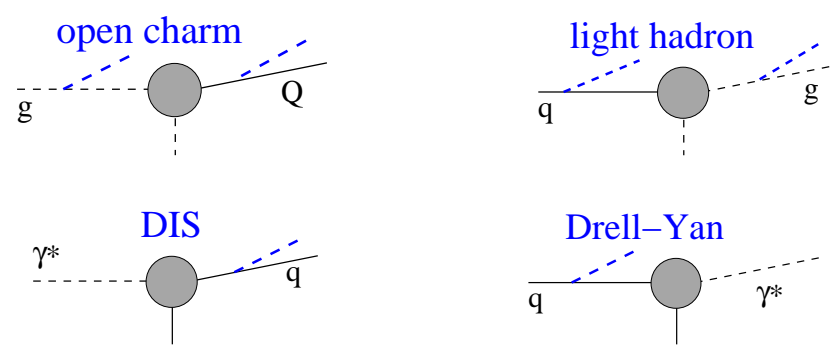

Figure 3: Partonic subprocesses where a medium-induced loss $\Delta E \propto E$ arising from coherent radiation is expected (top) or not (bottom). 


\section{Simple model for $J / \psi$ nuclear suppression}

Our model consists in expressing the $J / \psi$ differential production cross section $\mathrm{d} \sigma / \mathrm{d} x_{F}$ in pA collisions simply as that in $\mathrm{p}-\mathrm{p}$ collisions, with a shift in $x_{F}$ accounting for the energy loss $\varepsilon$ incurred by the octet $c \bar{c}$ pair propagating through the nucleus,

$$
\frac{1}{A} \frac{\mathrm{d} \sigma_{\mathrm{pA}}^{J / \psi}}{\mathrm{d} x_{F}}\left(x_{F}\right)=\int_{0}^{\varepsilon_{\max }} \mathrm{d} \varepsilon \mathscr{P}(\varepsilon) \frac{\mathrm{d} \sigma_{\mathrm{pp}}^{J / \psi}}{\mathrm{d} x_{F}}\left(x_{F}+\delta x_{F}(\varepsilon)\right),
$$

where $x_{F}(E)+\delta x_{F}(\varepsilon)=x_{F}(E+\varepsilon)$. At large enough $x_{F}$ we have $E \simeq x_{F} E_{\mathrm{p}}$ and $\delta x_{F}(\varepsilon) \simeq \varepsilon / E_{\mathrm{p}}$, where $E_{\mathrm{p}}$ is the incoming proton energy. The other ingredients in Eq. (3.1) are:

(i) The differential $\mathrm{p}-\mathrm{p}$ cross section, determined from a fit of $\mathrm{p}-\mathrm{p}$ data;

(ii) The energy loss probability distribution, or quenching weight $\mathscr{P}(\varepsilon)$, directly related to the medium-induced radiation spectrum $\mathrm{d} I / \mathrm{d} \omega$ associated to the hard subprocess (see Fig. 2) and derived in [9]. Since this spectrum is fully determined by $\Delta q_{\perp}^{2}=\hat{q} L$, the model depends on the single parameter $\hat{q}$.

In fact, $\hat{q}$ is related to the gluon distribution $G(x)$ in a target nucleon as $\hat{q}(x) \propto x G(x)$ [3]. Since $x G(x) \sim x^{-0.3}$ is suggested by fits to HERA data [11], $\hat{q}$ actually depends on $x=x_{2}$, where $x_{2}$ is the target parton momentum fraction,

$$
\hat{q}(x)=\hat{q}_{0}\left(\frac{10^{-2}}{x}\right)^{0.3} .
$$

Note that the $x$-dependence of $\hat{q}$ is not essential in our model. Similar final results would be obtained using a constant $\hat{q}(x)=\hat{q}$. In the following the single parameter of the model is taken as $\hat{q}_{0}=\hat{q}(x=0.01)$.

The model defined by Eq. (3.1) allows to predict the effects of parton energy loss on the nuclear attenuation factor (1.1). Besides energy loss effects, in the high energy domain under consideration we expect saturation of the nuclear gluon density at small $x_{2}$, leading to an additional $J / \psi$ suppression mechanism in $\mathrm{p}-\mathrm{A}$ collisions. The associated suppression is a scaling function of the saturation scale $Q_{s}$, which can be simply parameterized as [12]

$$
\mathscr{S}_{\mathrm{A}}^{\text {sat }}\left(x_{2}, L\right) \simeq \frac{a}{\left(b+Q_{s}^{2}\left(x_{2}, L\right)\right)^{\alpha}} .
$$

Note that the saturation scale $Q_{s}$ is directly related to the parameter $\hat{q}$ via $Q_{s}^{2}(x, L)=\hat{q}(x) L$ [13]. Thus, the inclusion of saturation effects does not require any additional parameter.

In order to make reliable predictions at RHIC and LHC, where saturation effects might be important, the $J / \psi$ nuclear production ratio will be determined assuming energy loss effects, $R_{\mathrm{pA}}^{\mathrm{E} . \text { loss }}$ from Eq. (3.1), with and without saturation effects,

$$
\text { (i) } \quad R_{\mathrm{pA}}=R_{\mathrm{pA}}^{\mathrm{E} \text {.loss }} \quad \text { (ii) } \quad R_{\mathrm{pA}}=R_{\mathrm{pA}}^{\mathrm{E} . \text { loss }} \times \mathscr{S}_{\mathrm{A}}^{\text {sat }} / \mathscr{S}_{\mathrm{p}}^{\text {sat }}
$$

\section{Results}

The parameter $\hat{q}_{0}$ is determined by the best fit provided by the model to the E866 fixed target data [4] for the ratio $R_{\mathrm{W} / \mathrm{Be}} \equiv R_{\mathrm{pW}} / R_{\mathrm{pBe}}$, see Fig. 4 (left). The fit gives $\hat{q}_{0}=0.09 \mathrm{GeV}^{2} / \mathrm{fm}$ assuming 
energy loss effects only, and $\hat{q}_{0}=0.05 \mathrm{GeV}^{2} / \mathrm{fm}$ when saturation effects are also included. The successful description of $J / \psi$ suppression in iron, $R_{\mathrm{Fe} / \mathrm{Be}}$ (Fig. 4 right), at the same energy fully supports the atomic mass dependence of the model.

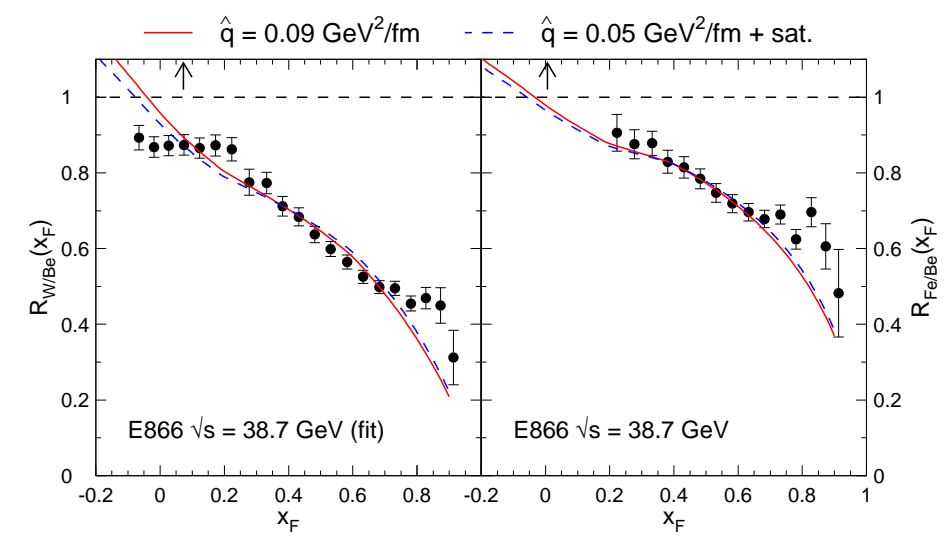

Figure 4: E866 $J / \psi$ suppression data [4] in $\mathrm{p}-\mathrm{W}$ (left) and $\mathrm{p}-\mathrm{Fe}$ (right) collisions compared to the energy loss model.

In Ref. [10], lower $\sqrt{s}$ (and smaller $x_{F}$ ) $J / \psi$ suppression data in p-A collisions (from NA3 [6], NA60 [14] and HERA-B [15]) are compared to the energy loss model, with the same value of $\hat{q}_{0}$. The agreement is very good, both in shape and magnitude, over a very wide range in $x_{F}$ [10]. The predictions including saturation are very similar to those without. As expected saturation effects are tiny at these energies.

The predictions in d-Au collisions at RHIC, $\sqrt{s}=200 \mathrm{GeV}$, are shown in Fig. 5 (left) in comparison with PHENIX data [7]. Energy loss effects are able to reproduce $J / \psi$ suppression at positive rapidities. Not surprisingly, some disagreement is observed in the negative $y$ bins for which nuclear absorption should play a role. Finally, arguing about the possible slight disagreement observed around mid-rapidity might be premature, given the present experimental uncertainties.
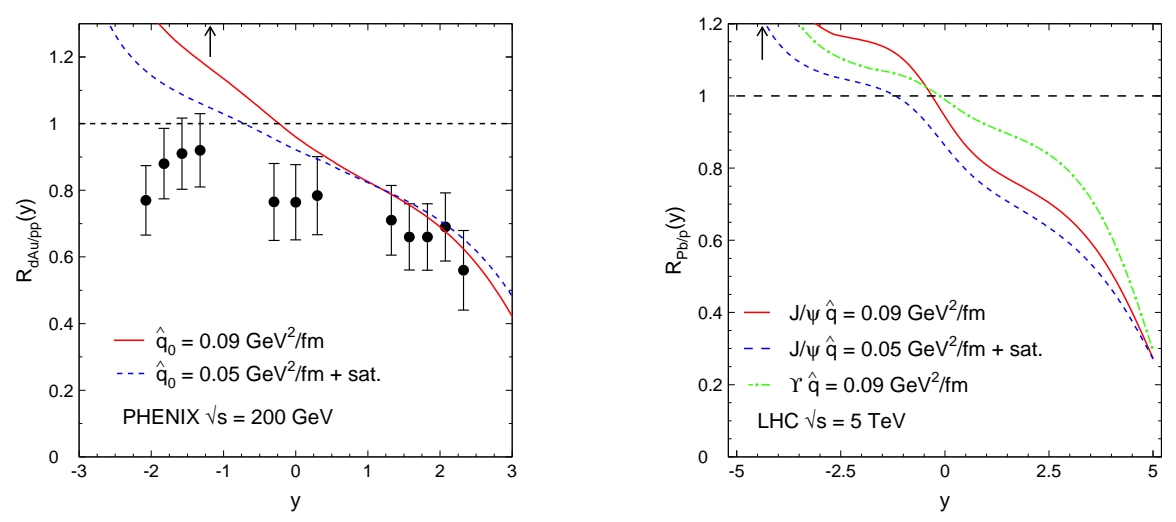

Figure 5: Left: PHENIX $J / \psi$ suppression data [7] in d-Au collisions compared to the energy loss model; Right: $J / \psi$ and $\Upsilon$ suppression expected in $\mathrm{p}-\mathrm{Pb}$ collisions at the LHC. 
Last, the rapidity dependence of $J / \psi$ suppression in $\mathrm{p}-\mathrm{Pb}$ collisions at the LHC (taking $\sqrt{s}=$ $5 \mathrm{TeV}$ ) is shown in Fig. 5 (right). Even though the inclusion of saturation effects is expected to yield a stronger $J / \psi$ suppression, it is somehow compensated by the use of a smaller transport coefficient; as a consequence $J / \psi$ predictions with (dashed line) and without (solid line) saturation are actually rather similar, except in the negative $y$-bins. Fig. 5 (right) also shows the predicted $\Upsilon$ suppression as a dash-dotted line. Because of the mass dependence of energy loss, $\Delta E \propto 1 / M[9$, $10]$, it is expected to be smaller than that of $J / \psi$ yet not negligible.

In summary, an energy loss model (supplemented by saturation effects) based on first principles has been presented. Once the transport coefficient $\hat{q}$ is determined from E866 data, the model is able to reproduce nicely all existing $J / \psi$ measurements in $\mathrm{p}-\mathrm{A}$ collisions. The studies of Refs. $[9,10]$ thus support parton energy loss as a dominant effect in p-A $J / \psi$ suppression. The model predictions for $J / \psi$ and $\Upsilon$ nuclear suppression at LHC energies can be compared to the future measurements by the ALICE and LHCb experiments during the $\mathrm{p}-\mathrm{Pb}$ run scheduled in 2012.

\section{References}

[1] G. Aad et al. [Atlas Collaboration], Phys. Rev. Lett. 105, 252303 (2010) [arXiv:1011.6182 [hep-ex]].

[2] S. Chatrchyan et al. [CMS Collaboration], Phys. Lett. B 712, 176 (2012) [arXiv:1202.5022 [nucl-ex]].

[3] R. Baier, Y. L. Dokshitzer, A. H. Mueller, S. Peigné and D. Schiff, Nucl. Phys. B 484, 265 (1997) [arXiv:hep-ph/9608322].

[4] M. J. Leitch et al. [FNAL E866/NuSea collaboration], Phys. Rev. Lett. 84, 3256 (2000) [arXiv:nucl-ex/9909007].

[5] S. S. Adler et al. [PHENIX Collaboration], Phys. Rev. Lett. 94, 082302 (2005) [arXiv:nucl-ex/0411054].

[6] J. Badier et al. [NA3 Collaboration], Z. Phys. C 20, 101 (1983).

[7] A. Adare et al. [PHENIX Collaboration], Phys. Rev. Lett. 107, 142301 (2011) [arXiv:1010.1246 [nucl-ex]].

[8] A. D. Frawley, T. Ullrich and R. Vogt, Phys. Rept. 462, 125 (2008) [arXiv:0806.1013 [nucl-ex]].

[9] F. Arleo, S. Peigné and T. Sami, Phys. Rev. D 83, 114036 (2011) [arXiv:1006.0818 [hep-ph]].

[10] F. Arleo and S. Peigné, arXiv:1204.4609 [hep-ph].

[11] K. J. Golec-Biernat and M. Wusthoff, Phys. Rev. D 59, 014017 (1998) [arXiv:hep-ph/9807513].

[12] H. Fujii, F. Gelis and R. Venugopalan, Nucl. Phys. A 780, 146 (2006) [arXiv:hep-ph/0603099].

[13] A. H. Mueller, Nucl. Phys. B 558, 285 (1999) [arXiv:hep-ph/9904404].

[14] R. Arnaldi et al. [NA60 Collaboration], Phys. Lett. B 706, 263 (2012) [arXiv:1004.5523 [nucl-ex]].

[15] I. Abt et al. [HERA-B Collaboration], Eur. Phys. J. C 60, 525 (2009) [arXiv:0812.0734 [hep-ex]]. 